\title{
Illusory form with inducers of opposite contrast polarity: Evidence for multistage integration
}

\author{
BIRGITTA DRESP, VÉRONIQUE SALVANO-PARDIEU, and CLAUDE BONNET \\ Université Louis Pasteur, Strasbourg, France
}

\begin{abstract}
The perception of brightness differences in Ehrenstein figures and of illusory contours in phaseshifted line gratings was investigated as a function of the contrast polarity of the inducing elements. We presented either continuous lines or line-likë arrangements composed of aligned dashes or dots whose spacing was varied. A yes/no procedure was used in which naive observers had to decide whether or not they perceived a brightness difference in a given Ehrenstein figure or an illusory contour in a phase-shifted line grating. The results show that brightness differences are perceived to some extent in Ehrenstein figures with inducers of opposite polarity of contrast; however, the percentage of yes responses was systematically lower and response times were longer than for figures with inducers of the same polarity. Phase-shifted line gratings with lines of opposite polarity of contrast yielded stronger illusory contours and shorter response times than those with lines of the same polarity. When the sign of contrast was not the same within a given line of induction, neither differences in brightness nor illusory contours were perceived. The results suggest that the mechanisms that lead to apparent differences in brightness are more sensitive to input of the same contrast polarity, the mechanisms generating illusory contours more sensitive to input of opposite polarity. The data are discussed in the light of a multistage approach to illusory form perception and some implications for cortical models of illusory contour integration are discussed.
\end{abstract}

Previous psychophysical investigations of illusory surfaces and contours have shown that local characteristics of the stimulus configuration determine the strength of the illusory percept that results from it. It has thus been established that the size and the spacing of inducing elements influence both global (Dresp, 1992; Dresp, Lorenceau, \& Bonnet, 1990; Watanabe \& Oyama, 1988) and local (Dresp \& Bonnet, 1991, 1993, 1995) sensitivity to illusory contours and brightness in the Kanizsa square. Similar size-spacing constraints seem to influence the magnitude of apparent brightness in the Ehrenstein figure (Zucker \& Davis, 1988) and the clarity of illusory contours in stimuli consisting of two phase-shifted gratings and other line configurations (Lesher \& Mingolla, 1993; Shipley \& Kellman, 1992). However, whether size and spacing factors more strongly affect the sharpness of the illusory contour or the brightness of the apparent surface has not been clearly determined, yet.

Some authors (e.g., Day \& Jory, 1980; Shapley \& Gordon, 1985, 1987; Ware, 1981) have emphasized the necessity of separating surface brightness from contour effects to obtain a deeper understanding of phenomena of illusory form. A phenomenal argument in favor of such a conceptual separation would be the fact that surface brightness and illusory contours, although they quite often

The authors would like to thank W. Ehrenstein, P. Kellman, L. Spillmann, and two anonymous reviewers for their helpful suggestions and comments on an earlier draft of this manuscript. Correspondence should be addressed to B. Dresp, Laboratoire de Psychophysique Sensorielle, Universite Louis Pasteur - C.N.R.S. 12, rue Goethe. 67000 Strasbourg, France (e-mail: bec $\omega$ currifl.u-strasbg.fr). occur together in one and the same stimulus, can occur separately in different figures. This observation inevitably suggests that, at some stage of processing, illusory surfaces and illusory contours are generated by independent mechanisms.

Another factor hitherto not systematically investigated in studies of illusory brightness and contour is the contrast polarity of the inducing elements. As a consequence, we still do not know the extent to which illusory brightness or contour integration is possible with inducers of varying contrast sign. It can, in particular, be expected that variations in contrast polarity do not affect illusory contours in the same way as they might affect illusory surface formation (Dresp, 1993; Prazdny, 1983; Shapley \& Gordon, 1987).

Neurophysiological models of illusory contour and brightness are constantly confronted with the problem of how to conceptually separate these two aspects of illusory form. Most of these models refer to receptive field properties of visual cortical cells as a key to understanding how information about an illusory line, or edge, is generated by combined activities of detectors that process orientation information provided by the inducing elements of the stimulus (Grossberg, 1994; Grossberg \& Mingolla, 1985; Heitger, Rosenthaler, Von der Heydt, Peterhans, \& Kübler, 1992; Lesher \& Mingolla, 1993; Peterhans \& Von der Heydt, 1989; Von der Heydt \& Peterhans, 1989).

An explicit distinction between brightness and contour effects is made in the model by Grossberg and colleagues. The authors assume that a multistage process involving hierarchically organized cortical mechanisms would account best for the genesis of illusory forms. At a first 
stage, the activities of detectors that are selective to the polarity of contrast (classically referred to as feature detectors) would generate local signals of differential brightness at line ends or other feature boundaries. These signals may then be averaged in space and give rise to a global representation of brightness differences within the figure. At a next stage, spatially distributed signals may be integrated via detectors that signal the presence of an illusory edge or contour, and that accept input of either polarity. The ideal illusory contour detector, as suggested by Grossberg and colleagues, would be an operator that integrates contrasts of any sign over the spatial scale defined by the size of its receptive field.

In our study, we wanted to determine the extent to which a hierarchical model of feature integration might successfully predict the perception of illusory surface brightness and contour in configurations with inducing lines of varying contrast sign. We used lines, aligned dashes, or aligned dots as inducers, and also varied the spacing between the stimulus elements. If hierarchical feature detection models based on the receptive field properties of visual neurons explain the genesis of illusory form, one may assume that the functional properties of the hypothetical detectors can be linked to specific perceptual responses. For example, brightness perception may be diminished, or abolished, when the inducing elements have opposite contrast signs. In this case, the local signals of differential brightness generated by the individual features should cancel each other out. As a consequence, no global representation of a brightness difference can be extracted from the stimulus. Illusory contour perception, on the other hand, may not be affected by variations in the contrast polarity of the inducing elements. Neurophysiological evidence tends to suggest that the cortical genesis of illusory contours involves all stages of processing, including mechanisms that integrate across polarities (e.g., Grosof, Shapley, \& Hawken, 1993; Heitger et al., 1992).

To provide separate accounts for effects on surface brightness on the one hand and effects on illusory contours on the other, we ran two experiments with different stimulus configurations and different observers. In both cases, we used line configurations in order to minimize differences in stimulus geometry. Generally, illusory forms induced by the ends of lines define one class of phenomena, and illusory forms induced by edges, as, for example, the well-known Kanizsa square, may be defined as another class of illusions, with presumably slightly different underlying mechanisms (see also Lesher \& Mingolla, 1993).

In our first experiment, the Ehrenstein figure (Figure 1a) was used to assess illusory figure lightness as a function of spacing and contrast polarity of the inducing lines. In this configuration, the shape of the illusory surface is ambiguous (see also Day \& Jory, 1980), and the observers can, if it is required, concentrate on global brightness differences without being influenced by a contour effect. In the second experiment, we used the socalled shifted grating illusion (see Figure $1 \mathrm{~b}$ ) to evaluate

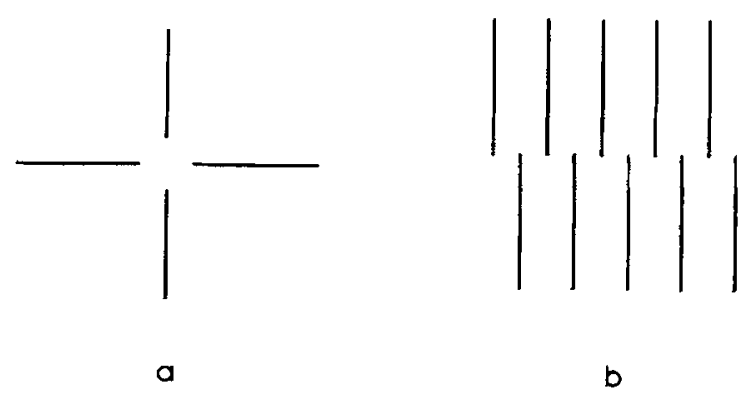

Figures 1a and 1b. The Ehrenstein figure (a) and the phase-shifted grating illusion (b). The first stimulus gives rise to the perception of apparent brightness enhancement in the center of the configuration. The illusory surface has no clearty defined boundaries. One may perceive a circle, a diamond, or a square in the center of the figure (see also Day \& Jory, 1980). In the second stimulus, one perceives a sharp, illusory contour separating the two phase-shifted gratings. There is no apparent diffenence in brightness between the two line surfaces.

illusory contour strength. In this figure, one perceives a sharp contour apparently separating two surfaces defined by sets of parallel lines, but no brightness difference between the surfaces.

\section{EXPERIMENT 1}

In the first experiment, observers had to decide whether or not they perceived a difference in brightness between the center of an Ehrenstein figure and its background, the luminance of both regions being strictly equal. The inducing figures consisted of either four continuous lines or four inducers made of either aligned dashes or aligned dots (see Figures 2, 2a, and 2b). The combinations of contrast polarities resulted in figures that appeared either lighter or darker than the background. The observers, therefore, were asked to decide on the presence or absence of a difference in lightness, knowing that it could be either positive or negative.

\section{Method}

Subjects. Ten observers participated in the experiment, ranging in age from 20 to 40 years. Some of them were familiar with illusory figures; others were not. All subjects, however, were naive to the purpose of the study. Two of them can be regarded as psychophysically trained. All observers had normal or corrected-to-normal vision.

Stimuli. The stimuli used in the first experiment are shown in Figures $2 a$ and $2 b$. They consisted of Ehrenstein figures with either four inducing lines or four inducers made of either aligned dashes or aligned dots. The polarity of contrast of the inducing elements could be uniform or alternating. In the case of uniform polarity, the inducers were either all black (as shown in Figure 2), or all white (not shown in the figure). In the configurations consisting of aligned dashes or dots, contrast polarity was varied both between and within inducing lines.

The luminance of the gray background upon which the stimuli were presented was $12 \mathrm{~cd} / \mathrm{m}^{2}$. The luminance of the white inducing elements was about $22 \mathrm{~cd} / \mathrm{m}^{2}$, and that of the black inducers about $2 \mathrm{~cd} / \mathrm{m}^{2}$. With these values, the strength of the illusion in the black version of a configuration with continuous lines appeared 

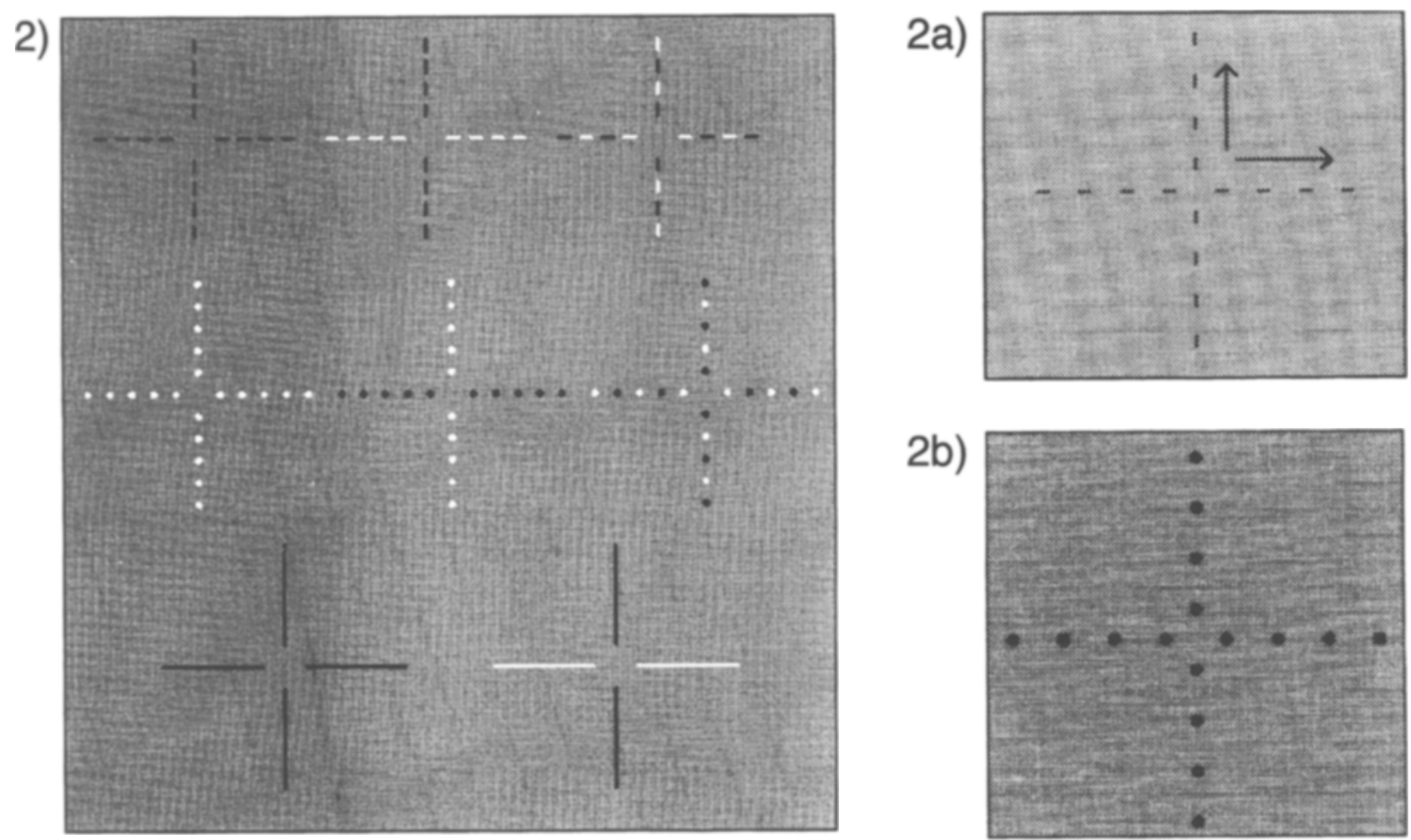

Figures 2, 2a, and 2b. Figure 2 shows the stimuli used in the first experiment. Ehrenstein figures made of continuous lines (bottom), aligned dashes (top), and aligned dots (middle) were presented to naive observers who had to judge as quickly as possible whether they penceived any difference in brightness between the figure and the ground. In configurations made of aligned dashes or dots the polarity of which varies within a given inducing line, the illusion is completely destroyed. In the stimuli with continuous lines, we varied the size of the central gap in the configuration; in the stimuli with dashes or dots, the central gap size was constant, and the space between individual dashes and dots was varied (2a and 2b).

equal to the strength produced by the white version. In figures with white inducers, the illusory region appeared darker; in stimuli with black inducers, it appeared lighter.

In the figures with continuous inducing lines, the size of the gap separating the lines was varied. Gap sizes were about $6,12,18,24$, and $30 \mathrm{arcmin}$. The width (about $2 \mathrm{arcmin}$ ) and the length of the lines (about $1^{\circ}$ ) were constant. In the figures made of aligned dashes or dots, the gap in the center of the configurations was held constant at 6 arcmin, and the space between dashes and dots was varied instead. Interelement spaces of $9,12,15,18$, and 38 arcmin were generated. The dots had a constant diameter of 2 arcmin, and the width (also 2 arcmin), and the length ( 4 arcmin) of the dashes in the other figures were also held constant. Altogether, 55 different stimulus configurations were presented to the 10 observers.

Procedure. The figures were generated by means of a VGATrident graphics card installed in a Hewlett-Packard Vectra 486. They were presented in random order on the computer screen (Philips Monochrome, 640*480,60 Hz), and each presentation was repeated four times within an experimental session consisting of 220 trials.

The observers were placed in front of the screen at a viewing distance of approximately $80 \mathrm{~cm}$ with their heads resting on a chin support. The figures appeared in the center of the screen and the experiment was run under low photopic viewing conditions. The subjects had to judge as quickly as possible whether or not the center of a given configuration appeared to differ in lightness compared to the background. The responses were encoded by means of a response key set connected to the computer. Response times were also computed. As soon as one of the two keys was struck, the figure disappeared from the screen and the next trial was initiated.
The time interval between stimulus offset and the presentation of the next figure was about $800 \mathrm{msec}$.

\section{Results and Discussion}

A first analysis consisted of testing whether the effects of white and black inducing elements in figures with only one contrast polarity (all inducing elements white, or all black) were, as we expected from the preliminary subjective strength adjustments, symmetrical. A global analysis of means revealed that the difference between the black and white conditions was indeed negligible and statistically nonsignificant for the two dependent variables (difference in percentage yes $<10 \%$, and difference in response time $<50 \mathrm{msec}$ ). A more detailed analysis showed that this held for figures made of continuous lines as well as for figures with dashes or dots. As a consequence, we averaged over these two contrast conditions and used the mean values for all further analyses.

A striking aspect of the data is that the figures made of dashes or dots the contrast polarity of which was alternating within a given inducing line did not engender the perception of lightness differences. In almost all cases, they yielded $0 \%$ of yes responses, and the response times showed only little variation (Figure $4 d$ ). The data indicate that, paradoxically, the figures with dashes gave rise to a slight increase, from $0 \%$ to $20 \%$ yes in the two con- 


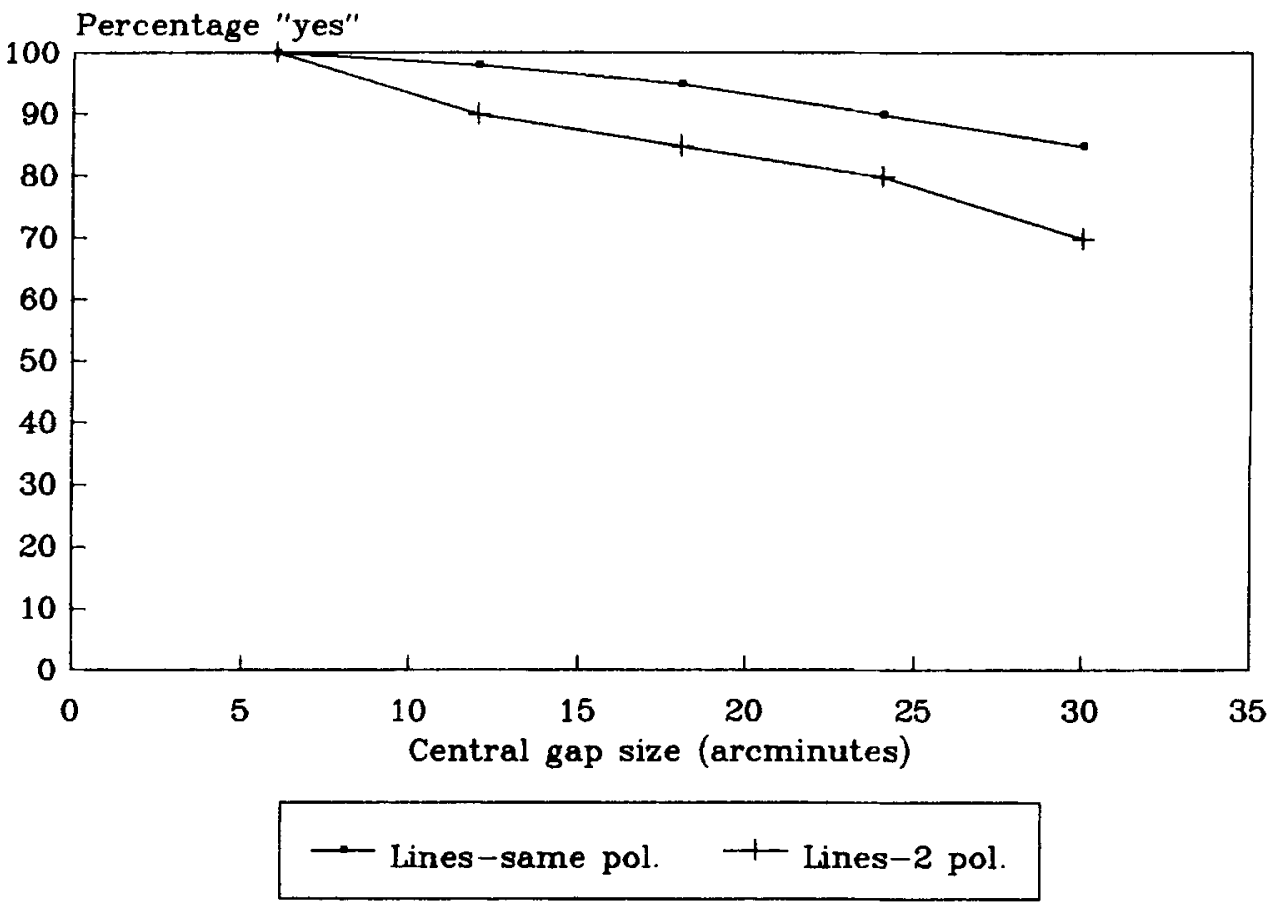

Figure 3a. The frequency of the perception of differences in brightness between the figure and the ground in stimuli made of continuous lines. The percentage of positive responses is plotted as a function of the size of the central gap in the figures, and the contrast polarity of the inducing elements (same vs. varying between inducing lines).

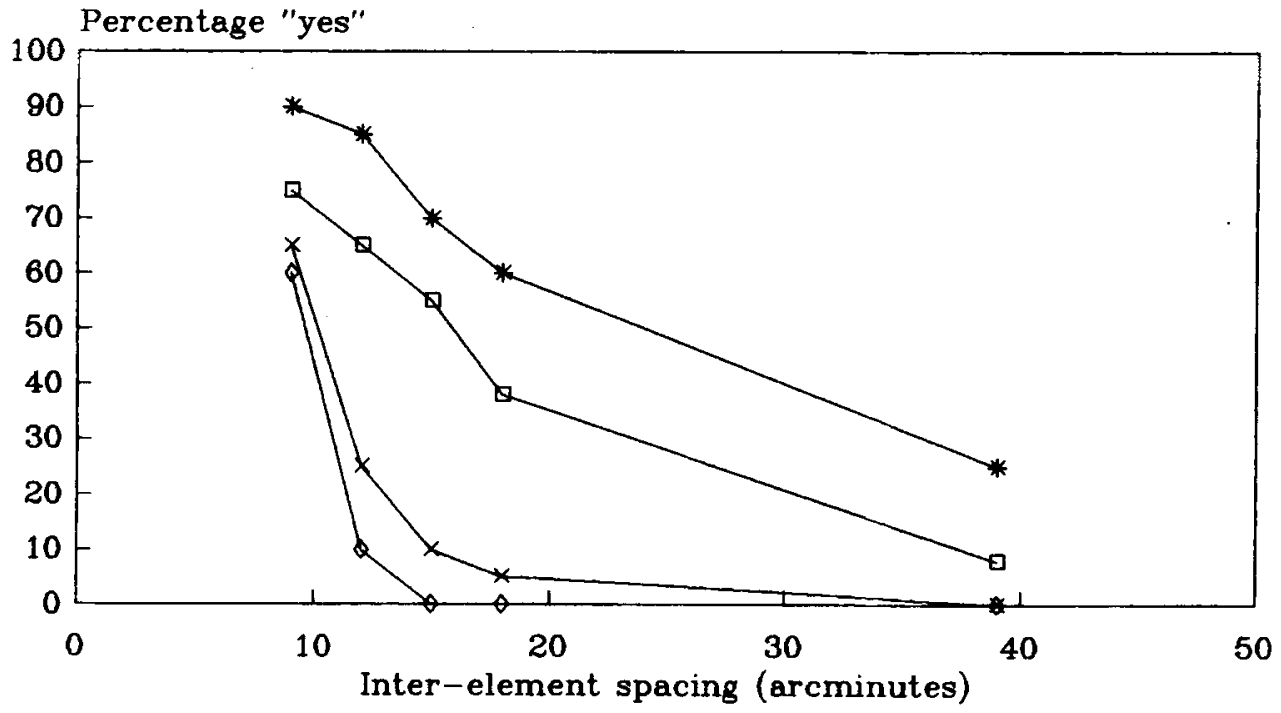

$$
\begin{array}{ll}
\text { * Dashes-same pol. } & \rightarrow \text { Dashes-2 pol. } \\
* \text { Dots-same pol. } & \rightarrow \text { Dots-2 pol. }
\end{array}
$$

Figure $3 b$. The frequency of the perception of differences in brightness in stimuli made of aligned dashes or dots. The percentage of positive responses is plotted as a function of the space between individual dashes or dots, and their contrast polarity (same vs. varying between inducing lines). 
ditions in which the spaces between the dashes were the largest (Figure 4d). A closer look at these stimuli suggests an explanation of this finding. In fact, when the gaps between the dashes become larger, a local configuration, appearing as a tiny Ehrenstein illusion induced by small, continuous lines of opposite contrast sign, emerges in the center of the figures. Given the particularity of these observations, we decided not to include the two stimulus conditions in which contrast polarity varied within the inducing lines in the global analysis of comparisons.

Global effects of contrast polarity and inducer type. In this analysis, we compared the results of the 10 observers in the two polarity conditions (same vs. varying between inducers) and the three figure conditions (continuous lines vs. dashes vs. dots) while averaging over the spacing factor. Generally, figures with inducing elements of the same contrast polarity were found to yield a stronger illusion, reflected through a higher percentage of yes responses, than figures with inducing elements of opposite contrast polarity $(61 \%$ yes and $40 \%$ yes, respectively). This effect was statistically significant $[F(1,18)=5.180$, $p<.05]$. Furthermore, response times were shorter for figures with elements of the same contrast polarity $(767 \mathrm{msec}$ compared to $863 \mathrm{msec})$. This effect was also statistically significant $[F(1,18)=6.854, p<.05]$.

Figures with continuous inducing lines produced significantly stronger illusions than figures with dashes, and figures with dashes did better than figures with dots $[90 \%, 58 \%$, and $18 \%$ yes, respectively; $F(4,72)=4.185$, $p<.01]$. Furthermore, response times were shorter for figures with lines than for figures with dashes, but slightly longer for stimuli with dashes than for stimuli with dots $(710,883$, and $780 \mathrm{msec}$, respectively). The effect of figure type on response time was not significant. Global interactions between contrast polarity and figure type were significant for neither of the two dependent variables.

The effect of interelement spacing was assessed in separate analyses for the three types of figures (figures with continuous lines, figures with dashes, and figures with dots). For each figure type, we compared the results of the 10 observers in the two polarity conditions (same vs. varying between inducers) and the five spacing conditions.

The results for figures with continuous inducing lines are shown in Figures $3 \mathrm{a}$ and $4 \mathrm{a}$. With increasing size of the gap in the center of the figures, the strength of the illusion decreased significantly $[F(4,72)=3.49, p<.025]$ and response times increased significantly $[F(4,72)=$ $7.916, p<.011$. As would be expected from the global comparisons, figures with lines of the same contrast polarity produced significantly stronger illusions $[F(1,18)=$ $4.83, p<.05]$ and shorter response times $[F(1,18)=5.63$, $p<.05]$. The interactions between polarity and spacing were nonsignificant. These data are represented in Figures $3 \mathrm{~b}$ and $4 \mathrm{~b}$. Apart from the observation that the figures made of aligned dashes produced generally weaker illusions than those obtained with configurations of continuous lines, the effects observed in both figure condi- tions are quite similar. Increasing the space between dashes had an effect comparable to that obtained when the size of the central gap in the figure varies. With increasing interelement spacing, the strength of the illusion decreased significantly $[F(4,72)=4.162, p<.01]$, while response times increased significantly $[F(4,72)=$ $6.88, p<.01]$. As would be expected from the global comparisons, figures with dashes of the same contrast polarity produced stronger illusions $[F(1,18)=12.264, p<$ $.01]$ and shorter reponse times $[F(1,18)=5.254, p<.05]$ than those with dashes the polarity of which varied from one inducing line to the other. As for figures with continuous lines, interactions between polarity and spacing were nonsignificant.

The configurations made of dots generally produced weak illusions, with low percentages of yes responses, falling rapidly to zero when interelement spacing increased (Figure $3 \mathrm{~b}$ ). The effect of interelement spacing on the percentage of yes responses was significant $[F(4,72)=$ $6.376, p<.01]$. In contrast to the other two figure conditions, response times decreased with increasing interelement spacing (Figure $4 \mathrm{c}$ ). This reversed effect of spacing on response time was significant $[F(4,72)=3.63$, $p<.025]$. This finding might have been due to the fact that the percentage of yes responses leveled out at zero in the three largest spacing conditions. In this case, the subjects should have been quite certain about the total absence of an illusion, and therefore their decision time would be expected to have been shorter than in conditions in which they were less certain about the total absence of an illusion and tended to respond yes in a certain proportion of the trials. As in the other figure conditions, stimuli with homogeneous contrast polarity produced stronger illusions than those with dots the polarity of which varied from one inducing line to the other $[F(1,18)=5.259$, $p<.05]$. In contrast to the other figure conditions, response times were longer for stimuli with homogeneous contrast polarity. Again, this reversed effect was significant $[F(1,18)=4.961, p<.025]$ and can also be explained on the basis of a high number of $0 \%$ yes responses in the conditions in which polarity was varying from one inducing line to the other. As in the other figure conditions, interactions between spacing and polarity were nonsignificant.

\section{Conclusions}

The results of this first experiment are consistent with earlier findings from studies using magnitude estimation or scaling techniques to evaluate the strength of illusory figures as a function of size-spacing constraints imposed by the inducing elements (Dresp et al., 1990; Shipley \& Kellman, 1992; Watanabe \& Oyama, 1988; Zucker \& Davis, 1988). They furthermore suggest that surface lightness/darkness in illusory figures similar to the one that is perceived in the Ehrenstein configuration can be expected to be stronger in configurations with inducers of the same contrast polarity, although stimuli in which polarity varies from one inducing line to the other to 


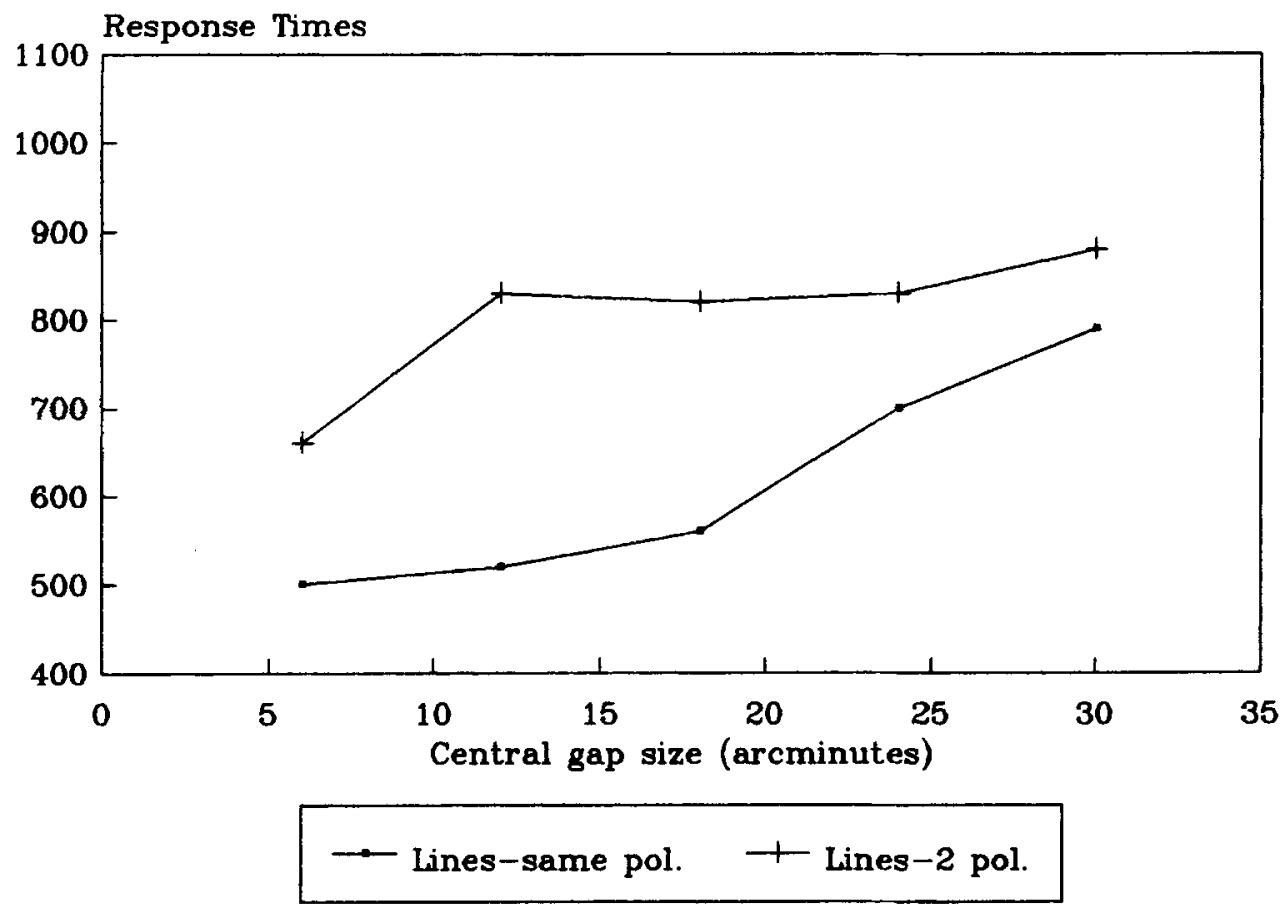

Figure 4a. Response times as a function of the spacing and the contrast polarity of the inducing elements for stimuli with continuous lines.

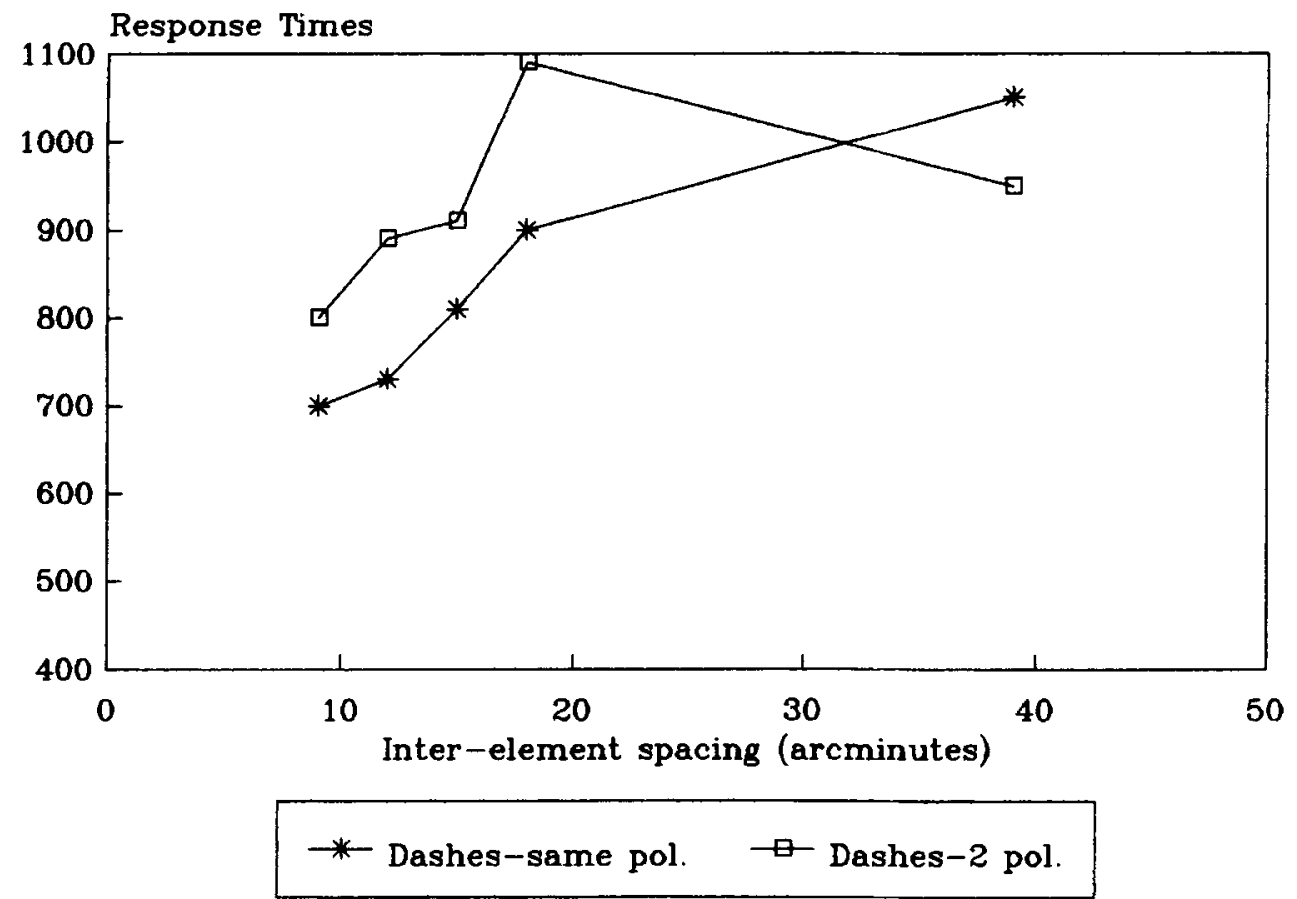

Figure $\mathbf{4 b}$. Response times as a function of the spacing and the contrast polarity of the inducing elements for stimuli with aligned dashes. 


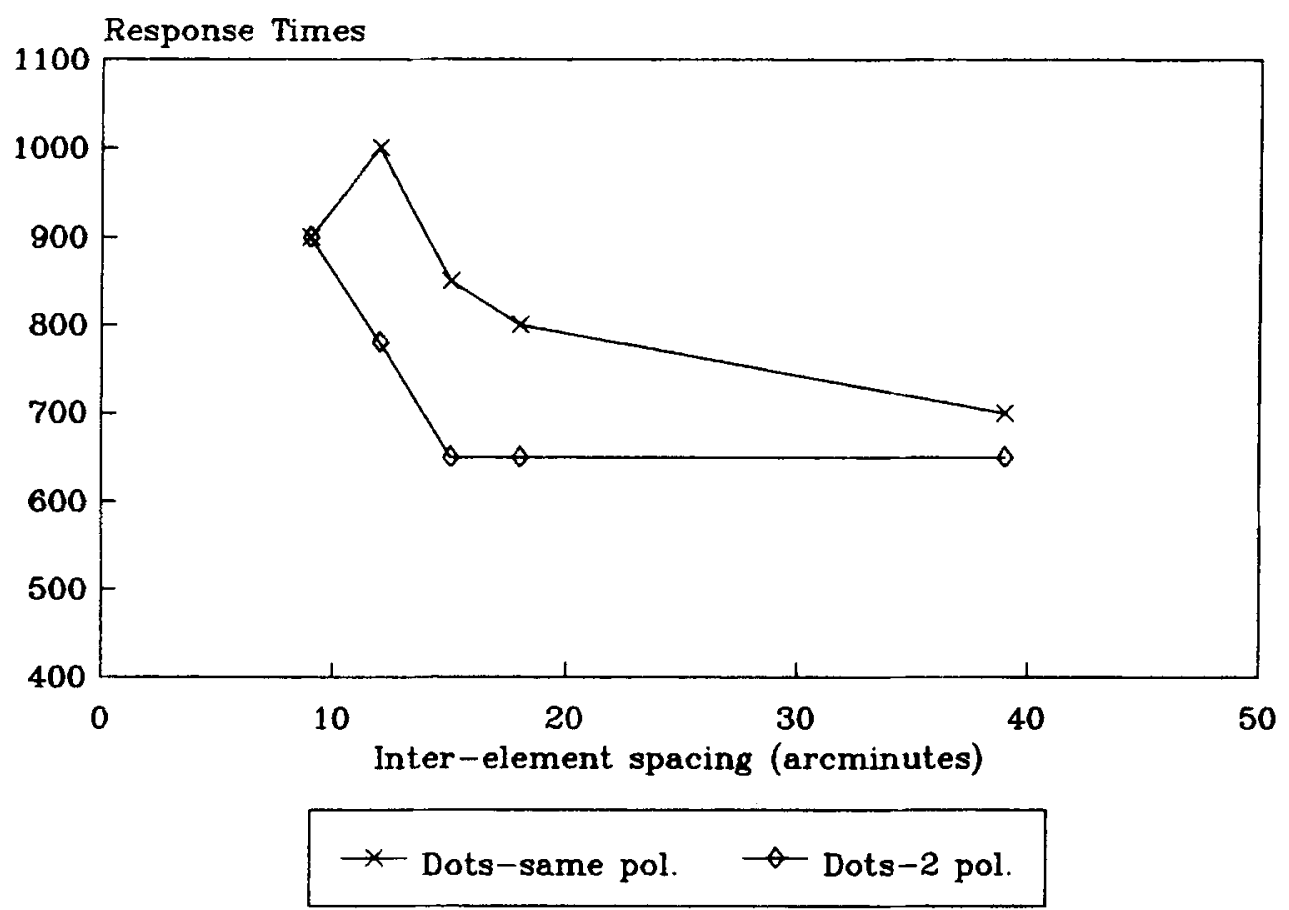

Figure $4 c$. Response times as a function of the spacing and the contrast polarity of the inducing elements for stimuli with aligned dots.

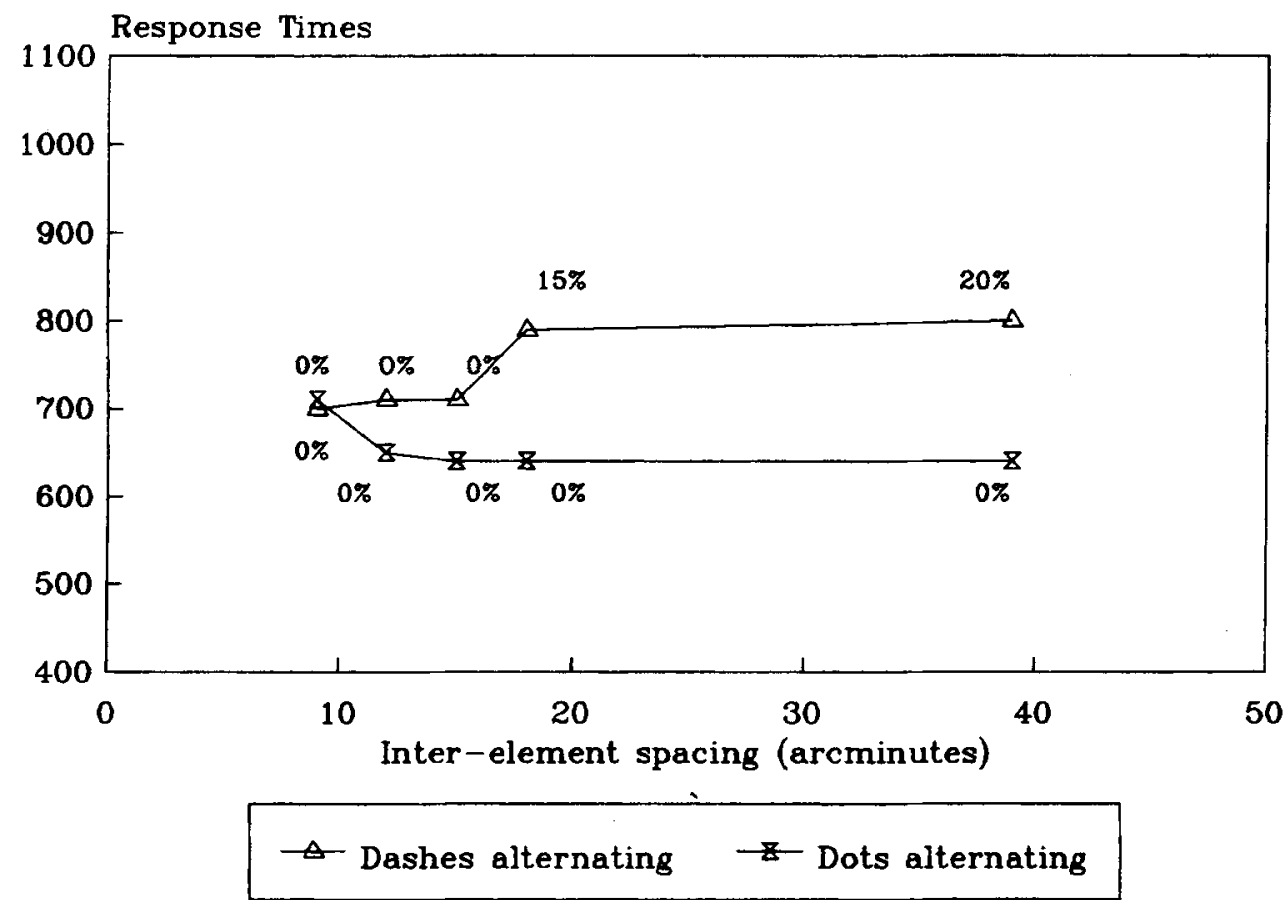

Figure 4d. Response times and frequency of the perception of brightness differences in figures made of aligned dashes or dots the polarity of which varies within inducing lines. The graphs show that these stimuli do not yield illusory percepts, and the response times vary hardly at all. 
some extent also seem to produce the surface enhancement. The process underlying this phenomenon can be described as one that integrates local and spatially discontinuous information. Collinear dashes seem to be integrated better than collinear dots. This suggests the existence of some kind of hierarchical organization in the process, with a critical sensitivity to local orientation. Finally, collinear inducing elements (dashes or dots) the polarity of which is not constant within a given line of induction fail to produce an illusory surface.

\section{EXPERIMENT 2}

In the second experiment, run with different observers to make sure that the responses would not be influenced by expectations from the first experiment, subjects had to decide whether or not they perceived an illusory contour in configurations consisting of two phase-shifted line gratings. As in the first study, the inducing elements were either continuous lines or line-like arrangements made of either aligned dashes or aligned dots (Figures 5, $5 \mathrm{a}$, and $5 \mathrm{~b}$ ). The contrast polarity of the inducers was varied as described above. However, with the phaseshifted grating stimuli, additional control of the global contrast of the two gratings was necessary. We took care that each side of a configuration contained the same number of white and black inducing lines to avoid differences in global contrast that could have artifactually enhanced the strength of illusory contours. Preliminary trials revealed that figures with dashes or dots the polarity of which varied within an inducing line (such combinations did not produce illusory brightness in the previous experiment) in no case yielded the perception of an illusory contour (Figure 5). Consequently, we did not include these stimulus conditions in our second experiment. Also, to make the sessions shorter for the subjects, we used only three spacing conditions in the second study.

\section{Method}

Subjects. Ten subjects, none of whom had participated in the first study, were run in this experiment. Some of them were familiar with illusory figures; others were not. All observers were naive to the purpose of the investigation and had normal or corrected-tonormal vision.

Stimuli. The stimuli used in this second experiment are shown in Figures $5 \mathrm{a}$ and $5 \mathrm{~b}$. The inducing lines were either continuous or made of aligned dashes or dots. The polarity of contrast of the inducing elements could be uniform or alternating. In the case of uniform polarity, the inducers were either all black (as shown in Figure 5), or all white (not shown in the figure). Background luminance, the luminance of inducing elements, and width and/or length were the same as in the first study. The orientation of the inducing lines was horizontal, and the phase-shifted line gratings thus produced vertical illusory contours. We varied the vertical gap separating the inducers in configurations made of continuous lines. Gap sizes were 12,18 , and 24 arcmin. In the configurations made of aligned dashes or dots, we held the vertical separation of the inducing lines constant at 12 arcmin, and varied the gaps between dashes and between dots. Gap sizes were 12, 15, and 18 arcmin.
Procedure. In this experiment, the observers had to judge, as quickly as possible, whether or not they perceived an illusory contour in a given stimulus configuration. The display and the general principles of the experimental procedure were the same as those in the first study.

\section{Results and Discussion}

As in the first experiment, the results obtained in the conditions in which the inducing elements all had the same contrast polarity (all black or all white) were symmetrical. Mean differences in the percentage of yes responses and in response time were negligible and statistically nonsignificant. Differences in the percentage of yes responses did not exceed $10 \%$, and differences in response time were smaller than $30 \mathrm{msec}$ in all figure conditions. As a consequence, we averaged over these two contrast conditions and took the mean values for all further comparisons.

As mentioned, to save time we did not include the figures in which contrast polarity varied within inducing lines in the second experiment. Preliminary trials on three naive observers had shown that these figures did not yield the perception of an illusory contour on any trial (for an illustration, see Figure 5).

Generally, the most striking finding in this second experiment, in which subjects had to decide on the presence or absence of an illusory contour, was that the effect of the contrast polarity of the inducers (same vs. varying between inducing lines) on both the strength of the illusion and the time necessary to respond to it, was found to be reversed compared to what was observed in the first study, in which subjects had to evaluate surface lightness/darkness.

Global effects of contrast polarity and inducer type. In the global analysis, we compared the results of the 10 observers in the two polarity conditions (same vs. varying between inducing lines) and the three figure conditions (figures with continuous lines vs. figures with dashed lines vs. figures with dotted lines) while averaging over the spacing factor. The data showed that, in general, illusory contours were more often perceived in configurations with inducers of opposite contrast polarity $(85 \%$ yes $)$ than in figures in which all inducing elements had the same contrast sign $(53 \%$ yes $)$. This effect was statistically significant $[F(1,18)=8.776, p<.01]$. Complementarily, response times were found to be significantly shorter when contrast polarity varied from one inducing line to the other $[1,090 \mathrm{msec}$ compared to $1,320 \mathrm{msec} ; F(1,18)=5.388, p<.05]$. As in the first experiment, figures with continuous lines yielded stronger illusions than figures with dashes, and dashes did better than dots $(93 \%, 64 \%$, and $49 \%$, respectively). The effect was significant $[F(2,36)=5.033, p<.25]$. Reponse times were generally shorter for figures made of continuous lines than for those made of dashed lines, and slightly longer for figures with dashes than for those with dots $(1,060,1,280$, and 1,270 msec, respectively). 
5)

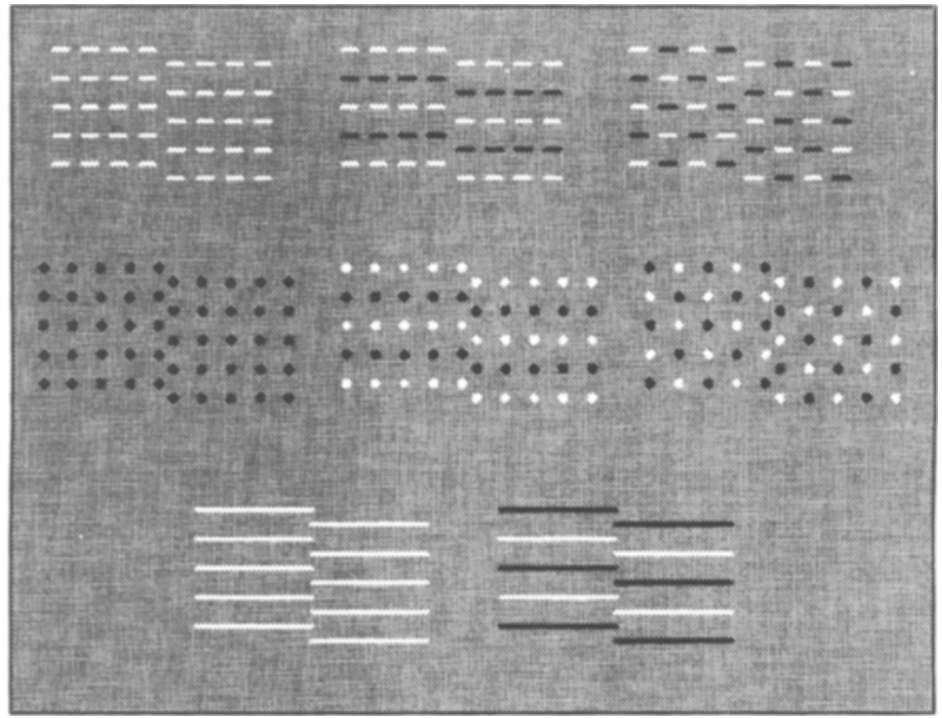

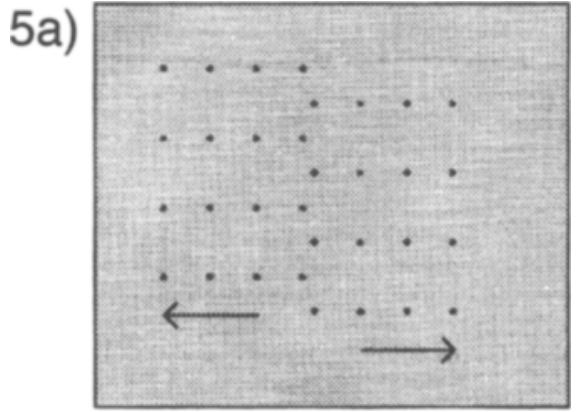

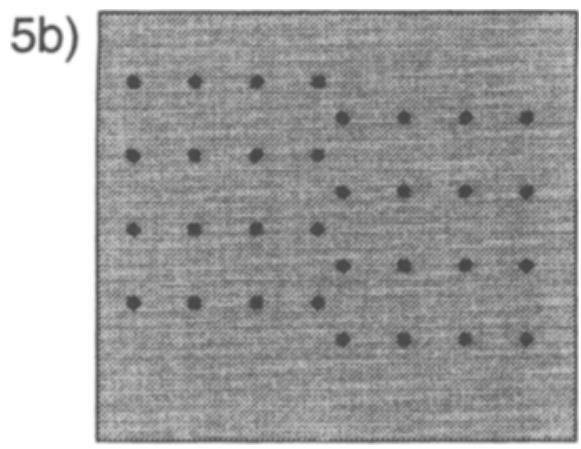

Figures 5, 5a, and 5b. Figure 5 shows the stimuli used in the second experiment. Phase-shifted line gratings made of continuous lines (bottom), aligned dashes (top), and aligned dots (middle) were presented vertically to naive observers who had to judge as quickly as possible whether they perceived an illusory contour in the stimulus or not. In configurations made of aligned dashes or dots the polarity of which varies within inducing lines, no illusory contour is perceived. After preliminary trials with 3 observers, we decided not to include these stimuli in the experiment to save some time. In the figures made of continuous lines, we varied the vertical space between inducing lines; in the figures made of dashes or dots, the vertical space between inducing lines was constant, and the space between individual dashes or dots varied ( $5 \mathrm{a}$ and $5 \mathrm{~b}$ ).

The effect of inducer type on response times was not significant, nor were interactions between polarity and inducer type.

As in the analysis of the data from the first experiment, we compared the results of the 10 observers in the two polarity conditions (same vs. varying between inducers) and the three spacing conditions separately for each type of figure. The results obtained with these figures are shown in Figures 6a and 7a. Gratings with lines of alternating contrast polarity yielded stronger illusions and shorter response times than gratings with lines of the same polarity $[F(1,18)=8.337, p<.01$, and $F(1,18)=$ $7.319, p<.025$, respectively]. Furthermore, when all lines had the same sign, the strength of the illusory contour slightly decreased when the lateral space between the inducing lines increased. This was not the case for lines with alternating polarity. The effect of line spacing on the percentage of yes responses was not statistically significant here, nor was the effect on response times, which varied only little (see Figure $7 \mathrm{a}$ ). This finding suggests that the limit of illusory contour integration was far from being attained with the lateral spacing sizes used in this experimental condition. Interactions between polarity and spacing were nonsignificant.

Gratings made of aligned dashes the polarity of which varied from one inducing line to the other produced stronger illusory contours and shorter response times than those with inducers of the same polarity $[F(1,18)=$ $9.31, p<.01$, and $F(1,18)=7.250, p<.25$, respectively; see Figures $6 \mathrm{~b}$ and $7 \mathrm{~b}]$. With increasing space between the dashes, the strength of the illusory contour decreased significantly $[F(2,36)=5.923, p<.01]$. Response times tended either to increase and then decrease again (gratings with inducers of the same contrast polarity; see Figure 7b) or to level out (gratings with inducers of opposite polarity). The effect of spacing on response times was not significant; nor were interactions between polarity and spacing.

As noted in the global analysis, figures with dots generally gave rise to the perception of an illusory contour less frequently than figures with dashes or continuous lines. As in the other figure conditions, however, stimuli in which contrast polarity varied from one inducing line to the other produced stronger illusory contours than stimuli in which all the dots had the same contrast polarity $[F(1,18)=6.093, p<.05$; Figure $6 \mathrm{~b}]$. In both cases, the percentage of yes responses decreased when the space between the dots increased $[F(2,36)=18.583, p<$ $.01]$. Although this decrease was slightly steeper for the figures in which all dots had the same contrast sign, the interaction between polarity and spacing was not significant. For figures with both polarities, response times first increased and then decreased again with increasing space between the dots (Figure 7c). When all dots had 


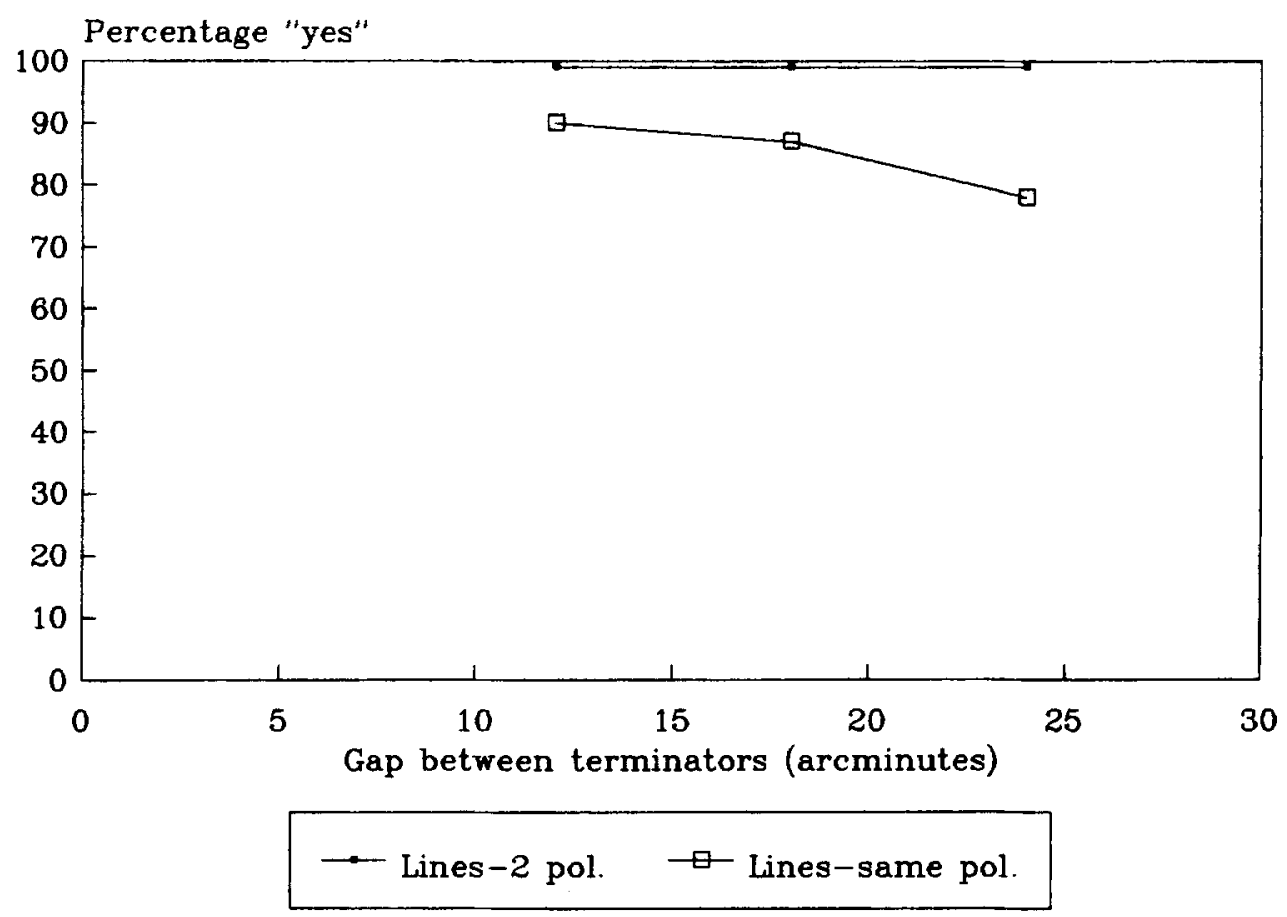

Figure 6a. The frequency of the perception of an illusory contour in figures made of continuous lines. The percentage of positive responses is plotted as a function of the size of the vertical gap between the inducing lines and their contrast polarity (same vs. varying between inducing lines).

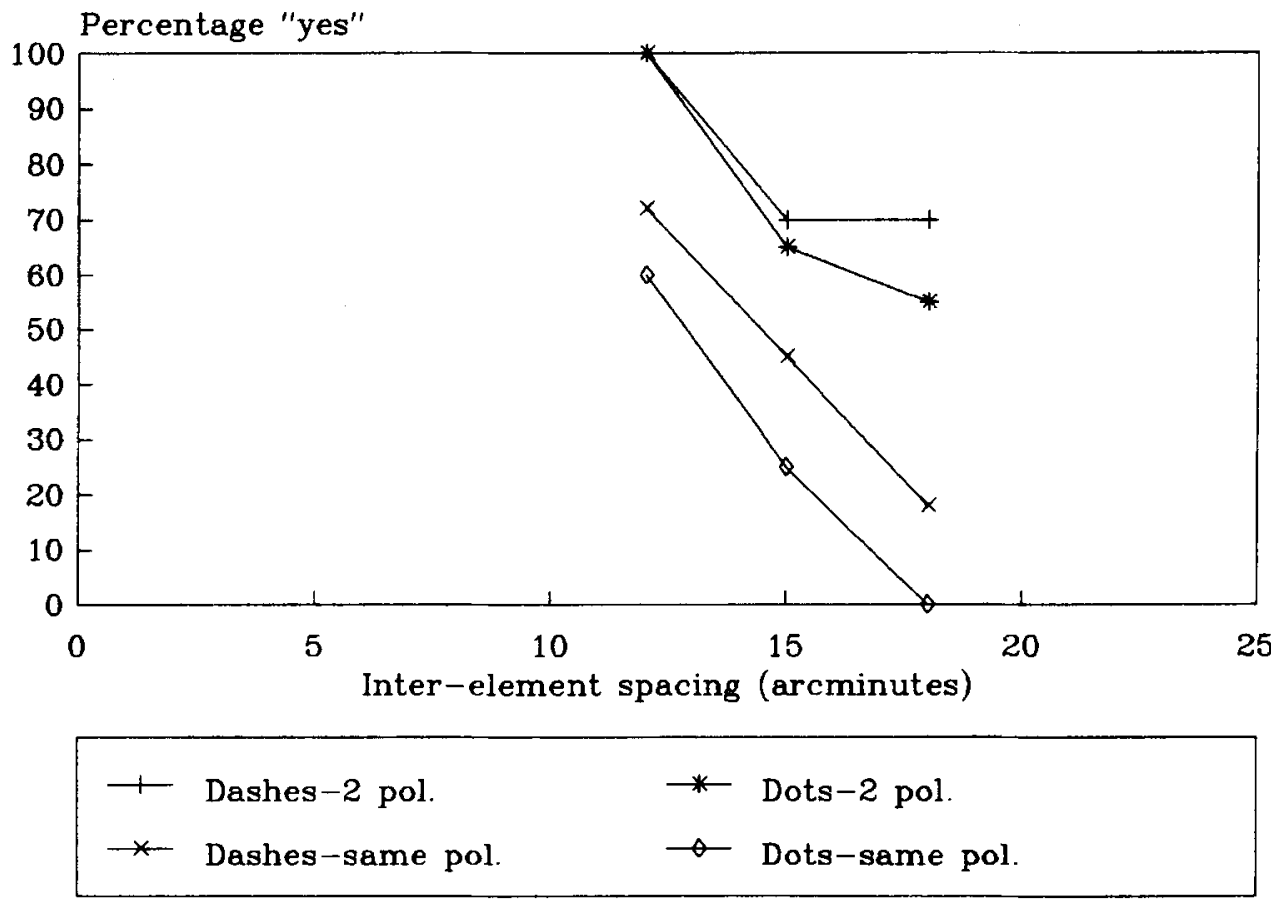

Figure $6 \mathrm{~b}$. The frequency of the perception of an illusory contour in configurations made of aligned dashes and dots as a function of their spacing and contrast polarity (same vs. varying between inducing lines). 

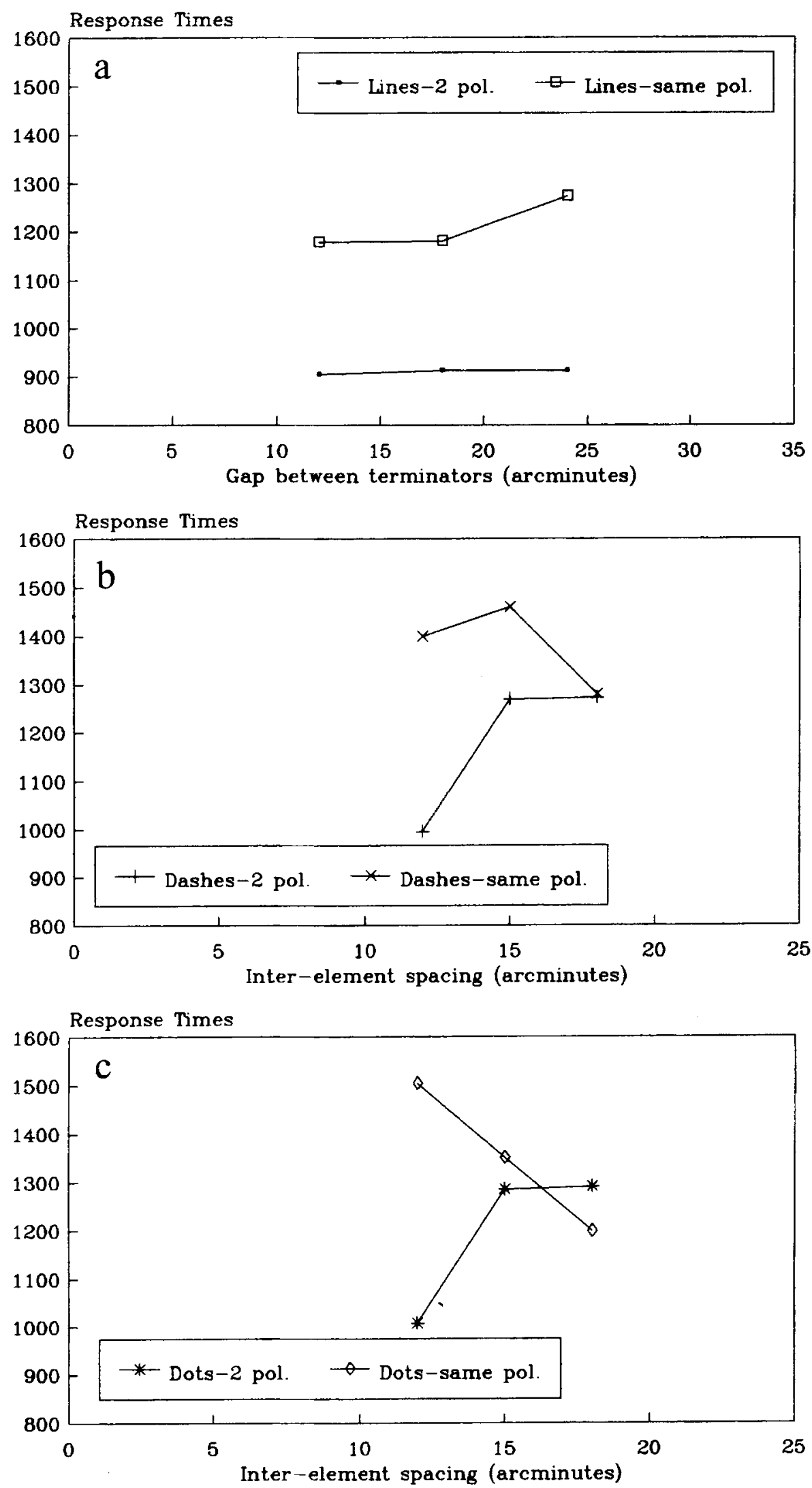

Figures $7 \mathrm{a}, 7 \mathrm{~b}$, and $7 \mathrm{c}$. Response times as a function of spacing and contrast polarity of the inducers for figures with continuous lines (7a), aligned dashes ( $7 b$ ), and aligned dots (7c). 


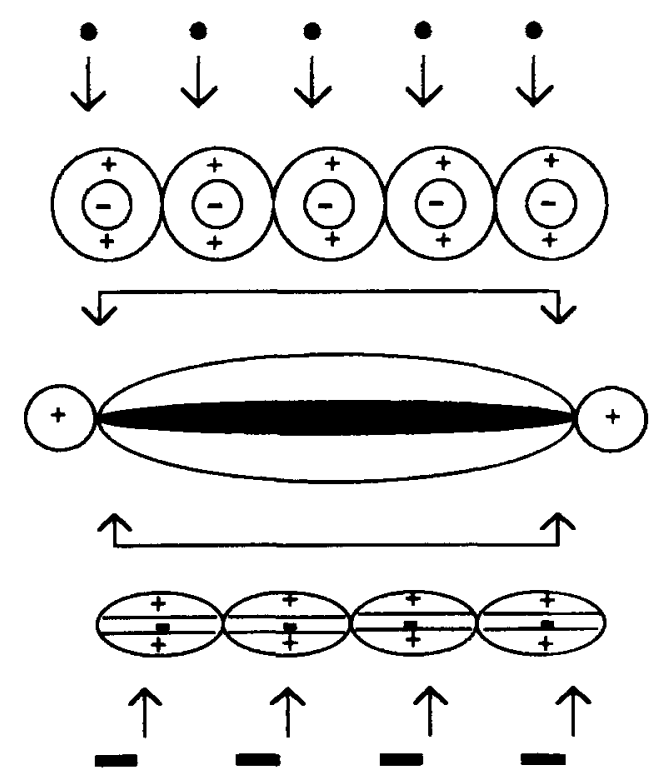

Figure 8. Figure 8 illustrates schematically how the integration of local signals triggered by individual stimulus elements such as dots or dashes may define the receptive field of a feature detector that generates signals of differential brightness. These signals, preferably when they are of the same sign (Experiment 1), would then input to the process responsible for the Ehrenstein illusion. End inhibition can explain how differential brightness is coded at the neural level (see, e.g., Peterhans, Von der Heydt, \& Baumgartner, 1986). When specific geometrical constraints are satisfied in a stimulus configuration (see, e.g., Kellman \& Shipley, 1991), summation or averaging of local brightness signals may generate surfaces (e.g., Grossberg, 1994).

the same contrast sign, response times steeply decreased with increasing space between the dots (in this case, the percentage of yes responses reached zero at the largest spacing value; cf. Figures $6 \mathrm{~b}$ and $7 \mathrm{c}$ ). The interaction between spacing and polarity was significant here $[F(2,36)=$ $3.488, p<.05]$.

\section{Conclusions}

The results of the second experiment confirm that the perception of an illusory contour depends on the same inducer size-spacing constraints as the perception of illusory surfaces (see Experiment I). In the case of spatial discontinuities within the inducing lines, collinear dashes were, again, found to produce better results than collinear dots. This suggests that local orientation is critical for illusory surfaces and contours. Collinear inducing elements the polarity of which is not constant within a given line of induction fail to produce illusory contours. The same conclusion has been found to hold for illusory surfaces (see Experiment 1).

However, the effect of variations in contrast polarity between inducing lines is reversed in the case of illusory contours. The data of the second experiment clearly show that illusory contours are better perceived in figures in which the contrast polarity varies from one inducing line to the other, whether the configuration is made of continuous lines, dashed lines, or dotted lines.

\section{GENERAL DISCUSSION}

The separate investigation of illusory surface brightness on the one hand and illusory contour strength on the other reveals that these two aspects of illusory form perception can, indeed, be functionally distinguished, as has been suggested earlier by Day and Jory (1980), Ware (1981), Shapley and Gordon (1985, 1987), and Kellman and Loukides (1987). Although both phenomena are found to depend in similar ways on the size-spacing constraints (see also Zucker \& Davis, 1988) imposed by the inducing elements of the illusory figure, they appear to be differently affected by local variations in contrast polarity.

Apart from the observation that both surface brightness (Experiment 1) and illusory contours (Experiment 2) are completely destroyed when the sign of contrast varies within inducing lines, it appears that integration of changes in contrast polarity from one inducing line to the other characterizes a critical step in the genesis of illusory contours (Experiment 2), whereas it seems to rather hinder the emergence of surface lightness or darkness (Experiment 1). Although the data from the first experiment tend to indicate that differences in surface lightness or darkness may also emerge to some extent from configurations with inducers of opposite contrast sign, we suspect that the phenomenon is different in nature in these cases. First, it takes significantly longer to be perceived and, furthermore, discussions with the observers revealed that they had difficulties in seeing neat lightness differences in Ehrenstein figures with inducers of opposite polarity. The subjects reported that they tended to infer the existence of such differences in these figures because the latter had sharp, diamond-shaped, illusory contours that did not emerge from the configurations with inducers of only one polarity. Thus, combining contrasts of different sign appears to strengthen the otherwise ill-defined figure boundaries (see Figure 9b, which will be discussed later).

The fact that configurations of lines with the same polarity yield more frequently and more rapidly the perception of differences in brightness between the figure and the background suggests that the underlying process prefers input of the same contrast sign. Thus, it may seem that a simple contrast model involving detectors of differential brightness would suffice to explain apparent brightness in the Ehrenstein illusion (see, e.g., Spillman, Fuld, \& Gerrits, 1976). However, the results of our first experiment indicate that local contrast cannot be the only critical factor. Although aligned dots of the same sign do produce the illusion, they have significantly less inducing power than continuous lines or aligned dashes. This observation strongly suggests that the process that generates the illusory surface must receive critical input from mechanisms that are sensitive not only to the contrast sign, but also to the orientation of local features. Cortical contrast mechanisms that integrate spatially discontinuous features within the spatial scale defined by their receptive fields provide a plausible model here (Figure 8). Some of the geometrical constraints that have 
a

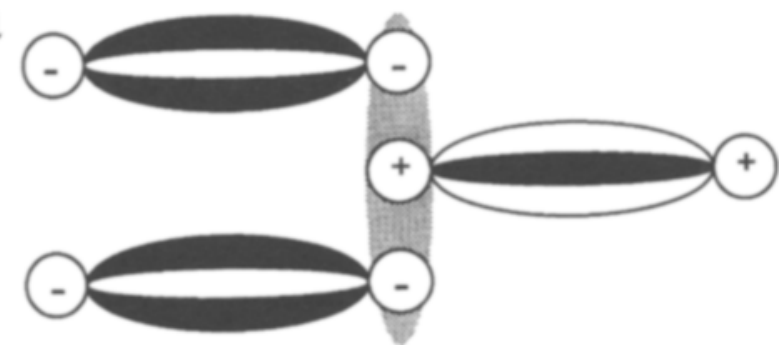

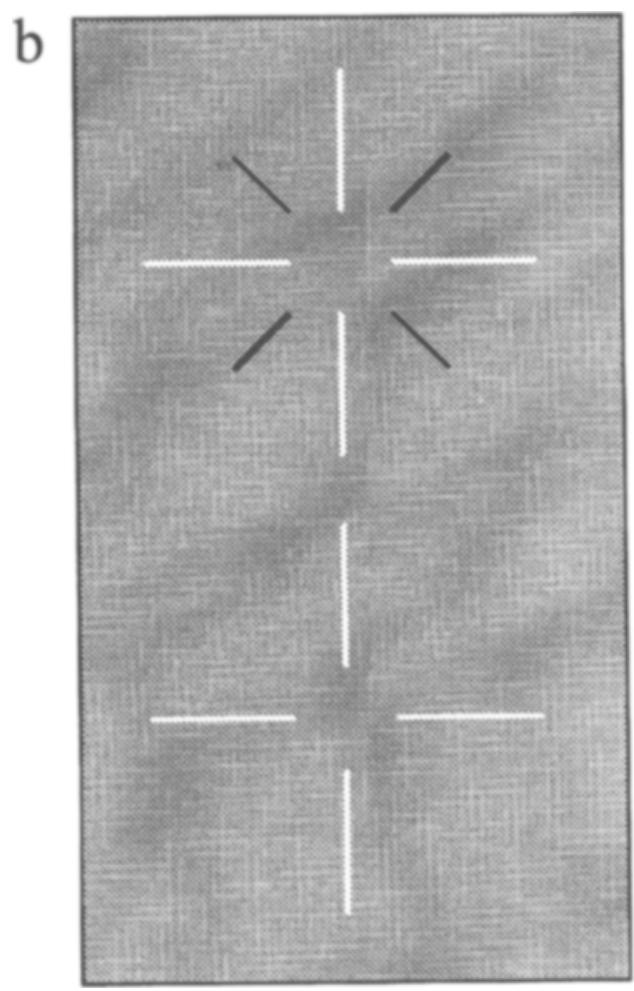

Figure 9a. Figure 9a illustrates schematically how the receptive field of an illusory contour detector may be built up on the basis of local signals of differential brightness. Integration of signals of opposite sign via detectors that are insensitive to the sign of contrast may explain why illusory contours are better perceived in configurations in which contrast polarity varies from one inducing line to the other (Experiment 2).

Figure 9b. A variation of Day and Jory's (1980) demonstration. This example shows how additional features of opposite contrast sign lead to the perception of a sharp illusory contour in a configuration that initially gives rise only to the perception of a fuzzy illusory area.

to be satisfied to establish perceptual links between individual features, namely line ends, in illusory figures have been formalized by Kellman and Shipley (1991).

How feature processing can explain apparent brightness or darkness has been simulated earlier on the basis of interactions between spatial filters (Zucker \& Davis, 1988), or by cortical network models involving detectors with the same orientational preference and position and receptive fields of different size, signaling brightness discontinuities at feature boundaries such as line ends (see, e.g., Grossberg, 1994; Grossberg \& Mingolla, 1985; Peterhans, Von der Heydt, \& Baumgartner, 1986). Summation or averaging of the signals would explain how global lightness or darkness enhancement is generated in various configurations. Obviously, there is for the moment no way of establishing a tighter link between any model of such a kind and experimental data as those shown here. This would be possible if we were in a position to provide local measures of brightness at distinct locations within the stimulus configurations, for example, by means of increment detection techniques. Earlier attempts in this direction failed to produce coherent results in the Ehrenstein figure (e.g., Spillman, Fuld, \& Neumeyer, 1984). As a consequence we must, for the time being, rely on psychophysical procedures that allow the quantitative assessment of global differences in brightness between the figure and the ground only.

The fact that illusory contours are significantly better perceived and take less time to emerge in configurations with inducers of opposite contrast sign suggests that their processing involves mechanisms that integrate across polarities fast, apparently over quite large distances. This conclusion is consistent with current knowledge on the neurophysiological correlates of illusory contours (Heitger et al., 1992; Peterhans et al., 1986) and implies that their genesis involves more complex cortical interactions than does the formation of apparent brightness. How the integration of signals of opposite contrast sign in the formation of illusory contours may work is illustrated in Figure $9 a$. The receptive field of an illusory contour detector, insensitive to contrast polarity, would be built up on the basis of spatial interactions between feature detectors that are polarity selective. This interpretation also explains why configurations of aligned dashes or dots the polarity of which varies within an inducing line do not yield illusory percepts of any kind (results of Experiments 1 and 2). In this case, the inducing lines do not stimulate polarity-selective feature detectors at the first stage, and 
further integration is therefore not possible. Such a conclusion is basically consistent with most of the current cortical models of illusory contour formation (see, e.g., Grossberg \& Mingolla, 1985; Peterhans \& Von der Heydt, 1989).

The idea that illusory contours may involve higher processes than the formation of apparent brightness was suggested earlier by Day and Jory (1980) on the basis of phenomenal demonstrations in the Ehrenstein illusion. The authors showed that adding dots at precise points in the figure can lead to the formation of clearly defined illusory contours. A variation of Day and Jory's demonstration is presented in Figure $9 b$, which shows that a strikingly sharp illusory contour emerges from the Ehrenstein configuration when lines of opposite contrast sign are placed at the otherwise ill-defined boundaries of the illusory surface.

In summary, the data from our two experiments suggest that configural effects on surface brightness and illusory contours (see also Kellman \& Loukides, 1987) involve mechanisms at different stages of cortical processing. They support the view that the perception of brightness enhancement on the one hand and the perception of illusory contours on the other ought to be functionally distinguished.

\section{REFERENCES}

DAY, R. H., \& JORY, M. K. (1980). A note on a second stage in the formation of illusory contours. Perception \& Psychophysics, 27, 89-91.

DRESP, B. (1992). Local brightness mechanisms sketch out surfaces but do not fill them in: Psychophysical evidence in the Kanizsa square. Perception \& Psychophysics, 52, 562-570.

DRESP, B. (1993). Bright lines and edges facilitate the detection of small light targets. Spatial Vision, 7, 213-225.

Dresp, B., \& Bonnet, C. (1991). Psychophysical evidence for lowlevel processing of illusory contours. Vision Research, 10, 18131817.

Dresp, B., \& BonNeT, C. (1993), Psychophysical measures of illusory form: Further evidence for local mechanisms. Vision Research, 33, 759-766.

DresP, B., \& BonNeT, C. (1995). Subthreshold summation with illusory contours. Vision Research, 35, 1071-1078.

DRESP, B., LoRENCEAU, J., \& BONNET, C. (1990). Apparent brightness enhancement in the Kanizsa square with and without illusory contour formation. Perception, 19,483-489.

Grosof, D. H., Shapley, R. M., \& Hawken, M. J. (1993). Macaque VI neurons can signal illusory contours. Nature, 365, 550-552.
Grossberg, S. (1994). 3-D vision and figure-ground separation by visual cortex. Perception \& Psychophysics, 55, 48-120.

Grossberg, S., \& Mingolla, E. (1985). Neural dynamics of form perception: Boundary completion, illusory figures, and neon color spreading. Psychological Review, 92, 173-211.

Heitger, F., Rosenthaler, L., Von der Heydt, R., Peterhans, E., \& KÜBLER, O. (1992). Simulation of neural contour mechanisms: From simple to end-stopped cells. Vision Research, 32, 963-981.

Kellman, P. J., \& Loukides, M. G. (1987). An object perception approach to static and kinetic subjective contours. In S. Petry \& G. E. Meyer (Eds.), The perception of illusory contours (pp. 151-164). New York: Springer-Verlag.

Kellman, P. J., \& SHIPley, R. (1991). A theory of visual interpolation in object perception. Cognitive Psychology, 23, 141-221.

Lesher, G. W., \& Mingolla, E. (1993). The role of edges and lineends in illusory contour formation. Vision Research, 33, 2253-2270.

Peterhans, E., \& Von der HeydT, R. (1989). Mechanisms of contour perception in monkey visual cortex: II. Contours bridging gaps. Journal of Neuroscience, 9, 1749-1763.

Peterhans, E., Von der Heydt, R., \& Baumgartner, G. (1986). Neuronal responses to illusory contour stimuli reveal stages of visual cortical processing. In J. D. Pettigrew, K. J. Sanderson, \& W. R. Levick (Eds.), Visual neuroscience (pp. 343-351). Cambridge: Cambridge University Press.

Prazdny, K. (1983). Illusory contours are not caused by simultaneous brightness contrast. Perception \& Psychophysics, 34, 403-404.

SHAPLEY, R., \& GoRDON, J. (1985). Nonlinearity in the perception of form. Perception \& Psychophysics, 37, 84-88.

Shapley, R., \& GoRDON, J. (1987). The existence of interpolated illusory contours depends on contrast and spatial separation. In S. Petry \& G. E. Meyer (Eds.), The perception of illusory contours (pp. $109-$ 115). New York: Springer-Verlag.

Shipley, T. F., \& Kellman, P. J. (1992). Strength of visual interpolation depends on the ratio of physically specified to total edge length. Perception \& Psychophysics, 52, 97-106.

Spillman, L., Fuld, K., \& Gerrits, H. J. M. (1976). Brightness contrast in the Ehrenstein illusion. Vision Research, 16, 713-719.

Spillman, L., Fuld, K., \& Neumeyer, C. (1984). Brightness matching, brightness cancellation, and increment threshold in the Ehrenstein illusion. Perception, 13, 512-520.

Von der Heydt, R., \& Peterhans, E. (1989). Mechanisms of contour perception in monkey visual cortex-I. Lines of pattern discontinuity. Journal of Neuroscience, 9, 1731-1748.

WARE, C. (1981). Subjective contours independent of subjective brightness. Perception \& Psychophysics, 29, 500-504.

Watanabe, T. \& Oyama, T. (1988). Are illusory contours a cause or a consequence of apparent differences in brightness and depth in the Kanizsa square? Perception, 17, 513-521.

Zucker, S. W., \& Davis, S. (1988). Points and end-points: A sizespacing constraint for dot grouping. Perception, 17, 229-247.

(Manuscript received June 13, 1994; revision accepted for publication June 19, 1995.) 